\title{
CREATIVE LIFE STORY WORK WITH SENIORS
}

\author{
PETRA BRANDOBUROVÁ \\ Centrum MEMORY, Mlynarovičova 21, 851 03, Bratislava, Slovakia \\ E-mail address: petra.brandoburova@gmail.com \\ SIMONA ADAMOVIČOVÁ \\ (c) $\doteqdot \circledast ㅇ$ \\ Centrum MEMORY, Mlynarovičova 21, 851 03, Bratislava, Slovakia \\ E-mail address: adamovicova.simona@gmail.com
}

\begin{abstract}
We summarize the theoretical background and basis for creative activities focused on the life story work with the seniors as realised in "Centrum MEMORY", Bratislava, Slovakia. Older adult clients with various levels of cognitive functioning could benefit from the combination of creative and reminiscence activities. The importance of creativity and its benefits in senior life is outlined and emphasized.
\end{abstract}

Key words: life story, seniors, reminiscence, creative activities

\section{INTRODUCTION}

"Centrum MEMORY" is the first preventative, diagnostic and specialized facility for people with dementia and memory impairment, one of its kind in terms of complexity of services in Slovakia. In its activities social services are being realised in daycare for people with dementia, psychodiagnostic services for people with memory impairment and educational activities for professionals caring for the persons with dementia of various etiology. The important part of "Centrum MEMORY" services are also various preventative or therapeutic programmes for clients, in which creative work with life stories is used .

\section{CREATIVITY IN LATER LIFE}

Creativity, the arts and cultural activities as part of a community is considered a human right (United Nations, 2013). Examples of how human creativity can drive progress, adaptation and innovation are well-known from the history and everyday life. What Mihaly Csikszentmihalyi (1999) points out in his system model about the creativity over a lifespan is that creativity is not a result of creativity test or the kind of judgement that somebody makes of our work. It is an idea 
or action that changes a cultural domain or something that could be more trivial (Csikszentmihalyi, 1990). Culture is made up by a multitude of domains, and when a domain is changed we have "Creativity". So a creative person is someone whose idea or action suceeds in changing one of the domains of culture. But we still have "creativity" which represents personal creativity - creativity in everyday life, that all of us are striving for and which will in some cases develop into cultural creativity. The creative process is what makes us totally human, and can make us happy despite all of the things that can go wrong in our lives including the period of old age. By understanding how the creativity works we have a chance to learn how to improve the quality of life for everyone including older adults (Csikszentmihalyi, 1995). The understanding of creativity in which we view all people as demonstrating a variety of creative characteristics and preferences, varying in degree and expression, is more recently presented by Donald Treffinger and his collborators (2012). Instead of asking how creative we are, they challenge us to move beyond looking at the level of creativity to the question how we are creative, considering the style of creativity, which could vary ways of expressing and its applying.

Creativity can be described in various terms and definitions. According to Carolyne Addams-Price (1998), it represents cognitive and emotional processes involved in the conception of meaningful products. Patsy Fasnacht (2003) defines it as the ability to recognize a problem and experiment with new ideas to solve the problem using divergent and convergent thinking. It can also be viewed as meta-cognitive process that generates novel and useful associations, attributes, elements, images, or sets of operations, and better solves a problem, produces a plan, or results in a product clearly not present before (Pesut, 1990). Creativity requires an individual to be open to new ideas and approaches, in order to seek an original solution for a problem or challenge at hand (Fisher, Specht, 1999). Problem-solving ability and creating novel products of outcome is basically the shared central characteristic in defining creativity. According to the investment theory of Robert Sternberg and Todd Lubart (1991), creativity requires a confluence of six distinct but interrelated resources: intellectual abilities, knowledge, styles of thinking, personality, motivation, and environment. Although levels of these resources are sources of individual differences, often the decision to use a resource is a more important source of individual differences. Creativity in this theory is in large part viewed as a decision, which suggests that creativity can be developed (Sternberg, 2006).

One of the popular current topics on creativity is aging creatively. As we age, many older adults might not feel themselves as being creative and their willingness to engage in creative activities is low. Society in general tends to have a negative view of aging and a belief that old age is a period of decline and older people no longer have the faculties or resources to respond creatively (Hickson, Housley, 1997). The negative view of ageing believes that old age brings a decline in ability to provide an original solution to a problem or challenge at hand (Formosa, 2013) and relative to younger adults, older adults 
typically behave more conventionally and are less likely to be open to new, original ideas (Zimprich et al, 2009). Factors which contribute to creative cognition such as problem solving, flexible thinking and memory retrieval could in fact decline with age. But the overgeneralized prejudice no doubt places its own restrictions on the expectations of the elderly themselves that they can maintain or even improve their creative abilities. The decreased cognitive capacity to be flexible and creative could reflect that older adults generally respond more conventionally because, compared to younger adults, they have a longer reinforcement history of conventional behavior and noncreative behavior is reinforced more often (Flora, 2004). Even in research the preconceived notions about aging and creativity prevent us from looking for more benign aspects of creativity in later life (Lindauer, 2003).

When exploring the relation between creativity and aging, two main models are available and dominated: the Peak and Decline model and the Life Span Developmental Model. In The Peak and Decline Model the degree of creativity is based on production, quantity and novelty. In accordance with that aging is viewed as the time of loss and creativity declines with aging (Frugé, 2016). Beginning in middle age and continuing into older adulthood, adults may experience declines in abilities related to creativity, such as the production of original solutions to open-ended problems (Reese et al., 2001). Alternatively Life Span Developmental Model holds the position that creativity and productivity are not equivalent and that creativity continues to grow, change and evolve with life span development (Reed, 2005). Observed declines in abilities related to creativity may not reflect an unavoidable consequence of aging, rather they may occur as a result of increasingly infrequent engagement in creative activities as individual age (Tranter, Koutstaal, 2008). Creativity is not a timebound act nor a function of chronological age and despite alternations in cognitive processing with age, it can be argued that the ageing brain is more adapt to imaginative thinking with increased distractibility and disinhibition (Hickson, Housley, 1997). The old age may simply represent a different kind of creativity and the ageing brain could be better suited to creative activity (Price, Tinker, 2014).

We offer the view that what is important is not the ability to generate a quantity of novel ideas and creations, but that the later life provides more time for reflection and construction of one's life story and the creativity should be supported in fulfilling this task. C. Addams-Price (1998) also concludes that late-life creativity reflects aspects of late-life thinking: synthesis, reflection, and wisdom. Therefore, in the alternative view as to how to evaluate the presence of creativity in older age, the individual's reflection, restructuring, and synthesis of ideas and wisdom could be characterized as creativity and similarly those interventions which stimulate these characteristics could be considered as creative activities (Flood, Phillips, 2007). What is mostly important is that being creative or to be involved in creative activity in older age can not only bring novel product or outcome, but also some other positive changes as the results of creativity. 


\section{IMPORTANCE OF CREATIVITY IN OLDER AGE}

Creativity plays an important role in the lives of older people promoting social interaction, providing cognitive stimulation and giving sense of self-worth (Price, Tinker, 2014). Through creativity, older people could potentially explore new endeavours, develop their sense of identity and cope better with the process of ageing (Fisher, Specht, 1999). Therefore, creative activity is one proposed coping mechanism for ageing individuals and populations. Developing creative coping strategies can enable the older adults to adapt more effectively to physical, psychological and psychosocial changes that occur during later life, increase adaptive skills and facilitate a flexible approach to handling everyday challenges (Flood, Phillips, 2007). Because of the physical and functional declines that are inevitable as one ages, greater creative ability would be a useful characteristic to have in order to more effectively adapt to inevitable age-related deterioration as well (Flood, Scharer, 2006).

Older age provides not only loses but also opportunities for growth through creative expression. Creative production throughout life's transitions can teach older adults much about themselves. It can help put into place the meaning of life and the meaning of aging, it may lead to both psychological and spiritual renewal and greater clarity of being. Creative flow may produce an optimal experience and can bring the fulfillment of producing even greater psychological growth and transformation (Hickson, Housley, 1997).

Through creative activity, the individual deepens an understanding of self and cultivates purposeful or meaningful involvements. Engagement in creative activities can provide both the opportunity to engage in independent decision making and the occasion to receive social reinforcement for doing so. This may result in an increase in independent behaviors and may also increase feelings of perceived environmental control (Hanneman, 2006). In this way, it strenghtens a sense of self that is competent, efficacious, and capable of doing (Fisher, Specht, 1999). The creative process benefits the older adult: to learn about oneself can lead to personal growth and to improvement of coping skills to more efficient ways of how to deal with the problems associated with the old age (Flood, Phillips, 2007). Findings also suggest some other potential benefits of creativity for senior health: mental clarity, improved emotional states and means for expressing experiences of life (Flood, Phillips, 2007). Further evidence of how creative engagement can contribute to healthy ageing can be found in the largest study of creativity to date which is Cohen's (2005) 3-year longitudinal study of creativity and ageing. The senior group with the implemented arts programming experienced diminished presence of problems and the use of medications, fewer doctor's visits and better scores on a geriatric depression scale and loneliness scale. 


\section{LIFE STORY METHODS}

Our creative life story work with seniors is based on biographical approaches, which provide older people with opportunity, if they so desire, to talk about their life experiences. A broad spectrum of life story methods can be identified in the domain of working with seniors which could be grouped in clusters such as reminiscence, life review, autobiography and life history. Robert Butler (1963) was the first to underscore the importance of reminiscence and life review in successful adaptation of older adults. He forwarded the idea that reviewing past plays an adaptive role in coming to terms with the finitude of the life. Reminiscence is the act or process of recalling and sharing one's personal memories and is distinguished from life review that also involves the evaluation of negative past experiences and conflicts. In order to fully apprehend the aging experience, it is necessary to listen to the stories that older people have to tell: the personal meaning of the life cycle is nowhere more clearly unfolded than in those who have clearly completed it (Westerhof, Bohlmeijer, 2014). Exploring older people's past and present lives with them, particularly the circumstances which have shaped their experiences, potentially provides greater insights into their needs and aspirations and may help to challenge ageist stereotypes about later life. Listening to a person's life story is a powerful way of showing that they are valued as an individual and may also have a cathartic value (Clarke, Hanson, Ross, 2003). Older age challenges us to sustain well being as we experience increased levels of vulnerability with factors such as changes in personal lifestyles, deterioration of physical health, loneliness etc.

It seems important to assist older people in developing coherent, meaningful life-story. The quest to derive from significance from years of lived experience is one of the most compelling challenges of aging. Experiences themselves do not have meaning until a person reflects on, recollects, and interprets them (Brady, Sky, 2003).

Reminiscence interventions have been developed specifically with this aim (Webster, Haight, 2002). Current promising development in reminiscence interventions include therapies in which reminiscence is combined with other approaches such as creative therapy (Willemse et al., 2009) or its simpler form creative reminiscence. Reminiscence integrated with creativity can increase the likelihood of self-transcendence as a critical component of wisdom and adaptation in later life and to help the older adult to organize challenges of life's events into some meaningful system (Levenson et al., 2005).

\section{CREATIVE REMINISCENCE}

Reminiscence is typically not considered as a creative activity, but it actually clearly demonstrates characteristics of creativity and can be realised using the art-based models of reminiscence. According to Marvin Formosa (2013), reminiscence entails cognitive-emotional processes of providing meaningful 
solutions to past problems. In his study of reminiscence sessions conducted with older adults, creative engagement of this form led to improved levels of personal empowerment. Firstly, it was by bringing about changes that enhance more positive attitudes towards life, better problem-solving skills, and greater emotional control (intrapersonal empowerment). Secondly, it was by stimulating changes in attitudes and skills related to interacting with other people, such as appreciation of other people's merits, willingness to seek help from others, and eagerness to care for others (interpersonal empowerment). Reminiscence as the life-review method can be further enhanced by creative expression of memories. In this way people are encouraged to create and discover metaphors, images and stories that symbolically represent the subjective and inner meaning of their lives (Bohlmeijer et al., 2005).

What we mean by creative reminiscence is encouraging people to explore their memories through creative activities which could be pleasurable for participants and entertaining for anyone with whom they may wish to share their results. Still to fulfill the quality of creative activity, it also should have three elements: innovation, valuable quality and appropriateness for the situation (Kaufman, Sternberg, 2007). Additional factors which should be taken into account include the notion that creative activities vary greatly between individuals, cultures and groups within society therefore the diversity of older population must be taken into account. The older population is diverse with the needs for older people ranging widely from active to those who are frail. Therefore creative reminiscence is beneficial because it involves a broad range of interests and can be encouraged in those with all functional abilities (Price, Tinker, 2014).

Reminiscence interventions are often only oral and that requires a good level of cognitive functioning to be preserved (Watt, Cappeliez, 2000). Reminiscence practices that rely primarily on memory, are mainly based on cognitive recall and can strengthen the experience of failure (Harries et al, 2013). The focus on broad themes like family history, accomplishments and turning points, which are often used in reminiscence, requires that people have the ability to review their life, to select the most important events, to summarize them and find a meaning in them (Bohlmeijer et al., 2005). For some older adults, this may be too complicated. We decided to use creative reminiscence as one of its greatest strengths is the number of ways it can be adapted so as to be appropriate for anyone, regardless of age or cognitive abilities. Creative expression which is the basic part of it is more appropriate not only for self-expression, but also as a way to access and explore memory. Arts-based approaches in reminiscence can offer a way to reflect and communicate non-verbally: therefore, older people can reminiscence about things they cannot or will not put into words. This allows older people to remember while at the same time containing their memories in an aesthetic form. Some older people may be better able or more willing to reminiscence under these circumstances (Larson, Perlstein, 2007). Virginia Batchelor (2009) stated that the main value of creative activity proces- 
ses is that it opens an emotional outlet, which in the case of dementia, when many cognitive functions are compromised, allows emotional expression, a key element for understanding the person with dementia. The explanation of why reminiscence activities can be successfully implemented for people with dementia is based on the memory functions distinction. Explicit memory is the form representing the direct and conscious recall or recognition of recently processed information. Implicit memory reflects the unconscious effects of previous experiences without conscious recollection. It is that type of memory, which can be relatively intact or less severely impaired in dementia memory impairment (Machado et al., 2009). According to Juhani Pallasmaa (2005), it is very natural to combine reminiscing with other activities that depend on procedural long-term memory - memories of embodied actions together with multi-sensory memory triggers as combined in creative reminiscence activities. He states that we remember through our bodies as much as through our nervous system and brain. Anne Davis Basting (2006) claims that people with dementia who have edited themselves into silence for fear of saying the wrong thing, or not understand, can use the arts to reconnect with themselves and the people who care for them. What she means by the arts is any medium used for creative expression - including creative reminiscence. According to her, the arts bring to people with dementia tools that enable them to express and their vision of the world and are particularly powerful for this group because the arts operate on an emotional level. Where rational language and factual memory have failed people with dementia, the arts offer an avenue for communication and connection with caregivers, loved ones, and the greater world - the arts offer a chance for people with dementia to connect with people who have forgotten them - their communities at large.

Engagement in creative activity and its components were also explored from the perspective of both clients and therapists in the study with elderly people dealing with life-threatening illness (la Cour et al., 2005). We consider the findings relevant for the creative reminiscence as it correspondents with our anecdotal experience when working with clients with dementia. This analysis indicated that creative activity was perceived in terms of multi-faceted core category of "creating connections to life", which was recognized as being made throughout the process of engaging in creative activity. Results should be interpreted with some attention to the possibility that the clients were disconnected before engagement in creative activity or that there was a need to reconnect or stay connected. The creation of connections in creative activity was provided by drawing up and refashioning memories and experiences from the client's life both therapists and participants in the activities re-established and supported their clients as individuals rather than in their roles as clients with disease. Furthermore, the engagement enabled clients to make connections between past experiences and their present situation and possible future. This kind of engagement holds potential for clients in relation with their actual abilities to confront and rebuild attachments to core cultural ideas and values as well as 
social relationships, not just with physically present people, but also remembered and idealized social others including their own past identities and self-images that are brought forward again to engage contemporary challenges.

\section{CONCLUSION}

Loss of the memory does not automatically mean loss of the creativity or capacity to feel and to express empathy. Music, movement, mime, touch, dance, drama and art can all be used, or used in conjunction with reminiscence (Gibson, 2011). Indeed it is essential that seniors with various cognitive functioning continue to have opportunities to express their creativity (Cohen, 2004). Creative reminiscence as a form of creative life story method might be beneficial to help older adults recognize the strengths they have and those that have carried them successfully during their ageing. It also provides an opportunity for self-expression building on own life experience. To feel comfortable looking forward the end of life, a person must come to terms with both the good and bad in life and allow for a final separation and acceptance of that history (Stephenson, 2006). Creative reminiscence includes the process of various creative actiivities (drawing, painting, music, movement etc.), in which concrete products are formed. The resulting objects serve afterwards as representative of the life story work and a point of discussion for curious others with whom the elder wishes to share.

\section{REFERENCES}

[1] Addams-Price, C. (1998). Creativity and successful aging. New York: Springer.

[2] Basting, A.D. (2006). Arts in Dementia Care. Generations, 30, 16-20.

[3] Batchelor, V. (2009). Creativity. In Birx, H. (Ed.), Encyclopedia of time: Science, philosophy, theology $\&$ culture. Thousand Oaks, CA: Sage Publications, pp. 238-244.

[4] Bohlmeijer, E. et al. (2005). Creative reminiscence as an early intervention for depression: Results of a pilot project. Aging \& Mental Health, 9(4), 302-304.

[5] Brady, E., \& Sky, H. (2003). Journal writing among older adults. Educational Gerontology, 29(2), 151-163.

[6] Butler, R.N. (1963). The life-review: An interpretation of reminiscence in the aged. Psychiatry, $26,65-76$.

[7] Clarke, A., Hanson, E.J., \& Ross, H. (2003). Seeing the person behind the patient: enhancing the care of older people using a biographical approach. Journal of Clinical Nursing, 12(5), pp. 697-706.

[8] Cohen, G. (2004). Uniting the Heart and Mind: Human Development in the Second Half of Life. A joint program of the American Society on Aging and MetLife Foundation.

[9] Cohen, G. (2005). The Mature Mind: The Positive Power of the Aging Brain. New York: Basic Books.

[10] Csikszentmihalyi, M. (1990). The domain of creativity. In Runco, M.A., \& Albert, R.S. (Eds.), Theories of creativity. Newbury Park: Sage Publications, pp. 190-212.

[11] Csikszentmihalyi, M. (1999). Creativity across the life-span: A systems view. In Colangelo, N., \& Assouline, S. (Eds.), Talent Development III. Great Potential Press, pp. 9-18.

[12] Fasnacht, P. (2003). Creativity: A refinement of the concept for nursing practice. Journal of Advanced Nursing, 42(2), 195-202. 
[13] Fisher, B., \& Specht, D. (1999). Successful aging and creativity later in life. Journal of Aging Studies, 13(4), 457-472.

[14] Flood, M., \& Phillips, K.D. (2007). Creativity in older adults: a plethora of possibilities. Issues in Mental Health Nursing, 28 (4), 389-411.

[15] Flood, M., \& Scharer, K. (2006). Creativity enhancement: possibilities for successful aging. Issues in Mental Health Nursing, 27(9), 939-959.

[16] Flora, S.R. (2004). The power of reinforcement. Albany: State University of New York Press.

[17] Formosa, M. (2013). Creativity in later life: possibilities for personal empowerment. In Hansen, A. et al. (Eds.), Creativity, lifelong learning and the ageing population.Ostersund: Jamtli Förla, pp. 78-91.

[18] Frugé, C.F. (2016). Creativity and Aging. [online], available: http://www.candacefaith.com/ upload/Creativity\%20and\%20aging.pdf [accessed 02/03/2016]

[19] Gibson, F. (2011). Reminiscence and Life Story Work. London: Jessica Kingsley Publishers.

[20] Hannemann, B.T. (2006). Creativity with dementia patients: Can creativity and art stimulate dementia patients positively? Gerontology, 52(1), 59-65.

[21] Harries, B. et al. (2013). The Storybox Project:Examining the Role of a Theatre and Arts-Based Intervention for People with Dementia. Manchester: University of Manchester.

[22] Hickson, J., \& Housley, W. (1997). Creativity in later life. Educational Gerontology, 23(6), 539-547.

[23] Kaufman, J.C., \& Sternberg, R.J. (2007). Resource review: creativity. Change: The Magazine of higher learning. [online], available: http://www.changemag.org/Archives/Back\%20 Issues/July-August\%202007/abstract-Resource-Review-Creativity.html [accessed 28/02/2016]

[24] la Cour, K. et al. (2005). Creating connections to life during life-threatening illness: Creative activity experienced by elderly people and occupational therapists. Scandinavian Journal of Occupational Therapy. 12(3), 98-109.

[25] Larson, R.T.H., Perlstein, S. (2007). When words are not enough: art-based methods of reminiscence. In Kunz, J.A., Gray Soltys, F. (Eds.), Transformational Reminiscence: Life Story Work. New York: Springer Publishing Company, pp. 123-142.

[26] Levenson, M. et al. (2005). Self-transcendence: Conceptualization and measurement. International Journal of Aging and Human Development, 60 (2), 127-143.

[27] Lindauer, M. 2003. Aging, Creativity and Art: A Positive Perspective on Late-Life Development, New York: Springer Publishing Company.

[28] Machado, S. et al. (2009). Alzheimer's disease and implicit memory. Arquivos de Neuro-Psiquiatria, 67 (2), 334-342.

[29] Pallasmaa, J. (2005). The Eyes of the Skin. Chichester: John Wiley and Sons.

[30] Pesut, D. (1990). Creative thinking as a self-regulatory metacognitive process: A model for education, training, and further research. Journal of Creative Behavior, 24(2),105-110.

[31] Price, K.A., \& Tinker, A.M. (2014). Creativity in later life. Maturitas, 78(4), 281-286.

[32] Reed, I. C. (2005). Creativity: Self-perceptions over time. International Journal of Aging and Human Development, 60 (1), 1-18.

[33] Reese, H.W. et al. (2001). Effects of intellectual variables, age, and gender on divergent thinking in adulthood. International Journal of Behavioral Development, 25(6), 491-500.

[34] Sternberg, R.J. (2006). The Nature of Creativity. Creativity Research Journal, 18(1), 87-98.

[35] Sternberg, R.J., \& Lubart, T.I. (1991). An investment theory of creativity and its development. Human Development, 34(1), 1-31.

[36] Stephenson, R.Ch. (2006). Promoting Self-Expression Through Art Therapy. Generations, 1, 24-26.

[37] Tranter, L.J., \& Koutstaal, W. (2008). Age and flexible thinking: An experimental demonstration of the beneficial effects of increased cognitively stimulating activity on fluid intelligence in healthy older adults. Aging, Neuropsychology, and Cognition. 15(2), 184-207.

[38] Treffinger, D.J. et al. (2012). Creativity in the Person: Contemporary Perspectives. LEARNing Landscapes, 6(1), 409-419.

[39] United Nations (2016). The Universal declaration of Human rights.[online], available: http:/ / www.un.org/en/universal-declaration-human-rights/ [accessed 10/02/2016] 
[40] Watt, L. M., \& Cappeliez, P. (2000). Integrative and instrumental reminiscence therapies for depression in older adults: Interventions strategies and treatment effectiveness. Aging $\mathcal{E}$ Mental Health, 4(2), 166-177.

[41] Webster, J.D., \& Haight, B.K. (2002). Critical advances in reminiscence work: From theory to application. New York: Springer Publishing Co.

[42] Westerhof, G.J., \& Bohlmeijer, E.T. (2014). Celebrating fifty years of research and applications in reminiscence and life review: State of the art and new directions. Journal of Aging Studies, $29,107-114$.

[43] Willemse, B.M. et al. (2009). A creative reminiscence program for older adults with severe mental disorders: Results of a pilot evaluation. Aging \& Mental Health, 13(5), 736-743.

[44] Zimprich, D. et al. (2009). Openness to experience, fluid intelligence, and crystallized intelligence in middle-aged and old adults. Journal of Research in Personality, 43(4), 444-454. 